Military Technical College

Kobry Elkobbah,

Cairo, Egypt

May 29-31,2012 $6^{\text {th }}$ International Conference

on Mathematics and

Engineering Physics

(ICMEP-6)

\title{
TIME VARYING ROTATING DISK FLOW AND HEAT TRANSFER THROUGH A POROUS MEDIUM OF A NON NEWTONIAN FLUID WITH SUCTION AND INJECTION
}

\author{
Hazem Ali Attia \\ Department of Engineering Mathematics and Physics, \\ Faculty of Engineering, El-Fayoum University, \\ El-Fayoum-63514, Egypt
}

\begin{abstract}
The time varying flow of an incompressible viscous non-Newtonian fluid above an infinite rotating porous disk in a porous medium is studied with heat transfer. A uniform injection or suction is applied through the surface of the disk. Numerical solutions of the nonlinear partial differential equations which govern the hydrodynamics and energy transfer are obtained. The effect of the porosity of the medium, the characteristics of the non-Newtonian fluid and the suction or injection velocity on the velocity and temperature fields is considered.
\end{abstract}

Keywords: Rotating disk flow, heat transfer, porous medium, non-Newtonian fluid, uniform inflow, numerical solution.

\section{Introduction}

The study of the hydrodynamic flow due to an infinite rotating disk was carried by von Karman [1] who gave a formulation of the problem and then introduced his famous transformations which reduced the governing partial differential equations to ordinary differential equations. Cochran [2] obtained asymptotic solutions for the steady hydrodynamic problem formulated by von Karman. Benton [3] improved Cochran's solutions and extended the problem to the transient state. The steady state heat transfer from a rotating disk maintained at a constant temperature was first considered by Millsaps and Pohlhausen [4] for a variety of Prandtl numbers, then Sparrow and Gregg [5] solved the problem for any Prandtl number. Attia [6] extended the problem discussed in $[4,5]$ to the unsteady state in the presence of an applied uniform magnetic field. The steady flow of a non-Newtonian fluid due to a rotating disk with uniform suction was 
Military Technical College

Kobry Elkobbah,

Cairo, Egypt

May $29-31,2012$

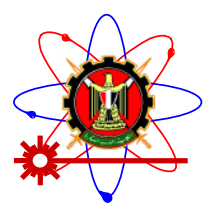

$6^{\text {th }}$ International Conference

on Mathematics and

Engineering Physics

(ICMEP-6)

considered by Mithal [7]. Then, Attia [8] extended the problem to the transient state with heat transfer. The study of a Newtonian fluid flow in a porous medium is studied by Attia [9] in the presence uniform suction and injection.

In the present paper, the time varying laminar flow through a porous medium of an incompressible viscous non-Newtonian fluid due to the uniform rotation of a porous disk of infinite extent is studied with heat transfer. A uniform injection or suction is applied through the surface of the disk. The flow in the porous medium deals with the analysis in which the differential equation governing the fluid motion is based on the Darcy's law which accounts for the drag exerted by the porous medium [10-11]. The governing nonlinear partial differential equations are solved numerically using the finite difference method. The effect of the porosity of the medium, the characteristics of the non-Newtonian fluid and the suction or injection velocity on the time varying flow and heat transfer is presented.

\section{Basic Equations}

Let the disk lie in the plane $z=0$ and the space $z>0$ is equipped by an incompressible viscous non-Newtonian fluid. The motion is due to the impulsive rotation from rest of an insulated disk of infinite extent about an axis perpendicular to its plane with constant angular speed $\omega$ through a porous medium where the Darcy model is assumed [10]. Otherwise the fluid is at rest under pressure $p_{\infty}$. The disk is maintained at a constant temperature $T_{w}$. A uniform injection or suction is applied at the surface of the disk for the entire range of suction or injection velocity.

The non-Newtonian fluid considered in the present paper is that for which the stress tensor $\tau_{j}^{i}$ is related to the rate of strain tensor $e_{j}^{i}$ as [7],

$\tau_{j}^{i}=2 \mu e_{j}^{i}+2 \mu_{c} e_{k}^{i} e_{j}^{k}-p \delta_{j}^{i}, e_{j}^{i}=0$,

where $p$ is denoting the pressure, $\mu$ is the coefficient of viscosity and $\mu_{c}$ is the coefficient of cross viscosity. The equations of steady motion are given by 
Military Technical College

Kobry Elkobbah,

Cairo, Egypt

May $29-31,2012$ $6^{\text {th }}$ International Conference

on Mathematics and

Engineering Physics

(ICMEP-6)

$\frac{\partial u}{\partial r}+\frac{u}{r}+\frac{\partial w}{\partial z}=0$

$\rho\left(\frac{\partial u}{\partial t}+u \frac{\partial u}{\partial r}+w \frac{\partial u}{\partial z}-\frac{v^{2}}{r}\right)+\frac{\mu}{K_{1}} u=\frac{\partial \tau_{r}^{r}}{\partial r}+\frac{\partial \tau_{r}^{z}}{\partial z}+\frac{\tau_{r}^{r}-\tau_{\varphi}^{\varphi}}{r}$

$\rho\left(\frac{\partial v}{\partial t}+u \frac{\partial v}{\partial r}+w \frac{\partial v}{\partial z}+\frac{u v}{r}\right)+\frac{\mu}{K_{1}} v=\frac{\partial \tau_{\varphi}^{r}}{\partial r}+\frac{\partial \tau_{\varphi}^{z}}{\partial z}+\frac{2 \tau_{\varphi}^{r}}{r}$

$\rho\left(\frac{\partial w}{\partial t}+u \frac{\partial w}{\partial r}+w \frac{\partial w}{\partial z}\right)+\frac{\mu}{K_{1}} w=\frac{\partial \tau_{z}^{r}}{\partial r}+\frac{\partial \tau_{z}^{z}}{\partial z}+\frac{\tau_{z}^{r}}{r}$

where $u, v, w$ are the velocity components in the directions of increasing $\mathrm{r}, \varphi, \mathrm{z}$ respectively, $P$ is denoting the pressure, $\mu$ is the coefficient of viscosity, $\rho$ is the density of the fluid, and $K_{1}$ is the Darcy permeability [10-11]. We introduce von Karman transformations [1],

$u=r \omega F, v=r \omega G, w=\sqrt{\omega v} H, z=\sqrt{v / \omega} \zeta, p-p_{\infty}=-\rho v \omega P$

where $\zeta$ is a non-dimensional distance measured along the axis of rotation, $F, G, H$ and $P$ are non-dimensional functions of $\zeta$ and $t$, and $v$ is the kinematic viscosity of the fluid, $v=\mu / \rho$. With these transformations, Eqs. (1)-(4) take the form

$$
\begin{aligned}
& \frac{\partial H}{\partial \zeta}+2 F=0 \\
& \frac{\partial F}{\partial t}-\frac{\partial^{2} F}{\partial \zeta^{2}}+H \frac{\partial F}{\partial \zeta}+F^{2}-G^{2}+M F+\frac{1}{2} K\left(\left(\frac{\partial F}{\partial \zeta}\right)^{2}+3\left(\frac{\partial G}{\partial \zeta}\right)^{2}+2 F \frac{\partial^{2} F}{\partial \zeta^{2}}\right)=0 \\
& \frac{\partial G}{\partial t}-\frac{\partial^{2} G}{\partial \zeta^{2}}+H \frac{\partial G}{\partial \zeta}+2 F G+M G-K\left(\frac{\partial F}{\partial \zeta} \frac{\partial G}{\partial \zeta}-F \frac{\partial^{2} G}{\partial \zeta^{2}}\right)=0 \\
& \frac{\partial H}{\partial t}-\frac{\partial^{2} H}{\partial \zeta^{2}}+H \frac{\partial H}{\partial \zeta}+M H+\frac{7}{2} K \frac{\partial H}{\partial \zeta} \frac{\partial^{2} H}{\partial \zeta^{2}}+\frac{\partial P}{\partial \zeta}=0
\end{aligned}
$$


Military Technical College

Kobry Elkobbah,

Cairo, Egypt

May $29-31,2012$ $6^{\text {th }}$ International Conference

on Mathematics and

Engineering Physics

(ICMEP-6)

where $M=v / K_{1} \omega$ is the porosity parameter and $K$ is the non-Newtonian parameter.

The initial and boundary conditions for the velocity problem are given by

$t=0: F=0, G=0, H=0$,

$\zeta=0: F=0, G=1, H=S$,

$\zeta \rightarrow \infty: F \rightarrow 0, G \rightarrow 0, P \rightarrow 0$,

where $S=w_{o} / \sqrt{\omega v}$ is the uniform suction or injection parameter, which takes constant negative values for suction and constant positive values for injection, and $w_{o}$ is the vertical velocity component at the surface. The above system of Eqs. (5)-(7) with the prescribed boundary conditions given by Eq. (9) are sufficient to solve for the flow velocity. Equation (8) can be used to solve for the pressure distribution if required.

Due to the difference in temperature between the wall and the ambient fluid, heat transfer takes place. The energy equation without the dissipation terms takes the form [4-5];

$\rho c_{p}\left(\frac{\partial T}{\partial t}+u \frac{\partial T}{\partial r}+w \frac{\partial T}{\partial z}\right)-k\left(\frac{\partial^{2} T}{\partial z^{2}}+\frac{\partial^{2} T}{\partial r^{2}}+\frac{1}{r} \frac{\partial T}{\partial r}\right)=0$

where $T$ is the temperature of the fluid, $c_{p}$ is the specific heat at constant pressure of the fluid, and $k$ is the thermal conductivity of the fluid. The boundary conditions for the energy problem are that, by continuity considerations, the temperature equals $T_{w}$ at the surface of the disk. At large distances from the disk, $T$ tends to $T_{\infty}$ where $T_{\infty}$ is the temperature of the ambient fluid. In terms of the non-dimensional variable $\theta=\left(T-T_{\infty}\right) /\left(T_{w}-T_{\infty}\right)$ and using von Karman transformations, Eq. (10) takes the form;

$$
\frac{\partial \theta}{\partial t}-\frac{1}{\operatorname{Pr}} \frac{\partial^{2} \theta}{\partial \zeta^{2}}+H \frac{\partial \theta}{\partial \zeta}=0
$$


Military Technical College

Kobry Elkobbah,

Cairo, Egypt

May 29-31,2012

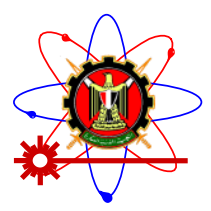

$6^{\text {th }}$ International Conference

on Mathematics and

Engineering Physics

(ICMEP-6)

where $\operatorname{Pr}$ is the Prandtl number, $\operatorname{Pr}=c_{p} \mu / k$. The initial boundary conditions in terms of $\theta$ are expressed as

$t=0: \theta=0$

$\theta(0)=1, \theta(\infty)=0$

The heat transfer from the disk surface to the fluid is computed by application of Fourier's law

$$
Q=-k\left(\frac{d T}{d z}\right)_{w}
$$

Introducing the transformed variables, the expression for $q$ becomes

$Q=-k\left(T_{w}-T_{\infty}\right) \sqrt{\frac{\omega}{v}} \frac{\partial \theta(t, 0)}{\partial \zeta}$

By rephrasing the heat transfer results in terms of a Nusselt number defined as, $N_{u}=Q \sqrt{\omega / v} / k\left(T_{w}-T_{\infty}\right)$ the last equation becomes

$N_{u}=-\frac{\partial \theta(t, 0)}{\partial \zeta}$

Numerical solution for the governing nonlinear Eqs. (5)-(7) with the conditions given by Eq. (9), using the finite-differences, leads to a numerical oscillation problem resulting from the discontinuity between the initial and boundary conditions (9a) and (9b). The same discontinuity occurs between the initial and boundary conditions for the energy problem (see Eq. (12)). A solution for this numerical problem is achieved using proper coordinate transformations, as suggested by Ames [12] for similar problems. Expressing Eqs. (5)-(7) and (11) in terms of the modified coordinate $\eta=\zeta / 2 \sqrt{t}$, we get

$$
\frac{\partial H}{\partial \eta}+4 \sqrt{t} F=0
$$


Military Technical College

Kobry Elkobbah,

Cairo, Egypt

May $29-31,2012$ $6^{\text {th }}$ International Conference

on Mathematics and

Engineering Physics

(ICMEP-6)

$$
\begin{aligned}
& \frac{\partial F}{\partial t}-\frac{\eta}{2 t} \frac{\partial F}{\partial \eta}-\frac{1}{4 t} \frac{\partial^{2} F}{\partial \eta^{2}}+\frac{1}{2 \sqrt{t}} H \frac{\partial F}{\partial \eta}+F^{2}-G^{2}+M F+\frac{K}{8 t}\left(\left(\frac{\partial F}{\partial \eta}\right)^{2}+3\left(\frac{\partial G}{\partial \eta}\right)^{2}+\right. \\
& \frac{\partial G}{\partial t}-\frac{\eta}{2 t} \frac{\partial G}{\partial \eta}-\frac{1}{4 t} \frac{\partial^{2} G}{\partial \eta^{2}}+\frac{1}{2 \sqrt{t}} H \frac{d G}{d \eta}+2 F G+M G-\frac{K}{4 t}\left(\frac{\partial F}{\partial \eta} \frac{\partial G}{\partial \eta}-F \frac{\partial^{2} G}{\partial \eta^{2}}\right)=0 \\
& \frac{\partial \theta}{\partial t}-\frac{\eta}{2 t} \frac{\partial \theta}{\partial \eta}-\frac{1}{4 t \operatorname{Pr}} \frac{\partial^{2} \theta}{\partial \eta^{2}}+\frac{1}{2 \sqrt{t}} H \frac{\partial \theta}{\partial \eta}=0 .
\end{aligned}
$$

Equations (13)-(16) represent a coupled system of non-linear partial differential equations which are solved numerically under the initial and boundary conditions (9) and (12), respectively using finite difference approximations. A linearization technique is first applied to replace the nonlinear terms at a linear stage, with the corrections incorporated in subsequent iterative steps until convergence is reached. The CrankNicolson implicit method is then used at two successive time levels [12]. An iterative scheme is used to solve the linearized system of difference equations. The solution at a certain time step is chosen as an initial guess for next time step and the iterations are continued till convergence, within a prescribed accuracy. Finally, the resulting block tridiagonal system is solved using the generalized Thomas-algorithm [12]. Finite difference equations relating the variables are obtained by writing the equations at the mid point of the computational cell and then replacing the different terms by their second order central difference approximations in the $\eta$-direction. The diffusion terms are replaced by the average of the central differences at two successive time-levels. The computational domain is divided into meshes each of dimension $\Delta t$ and $\Delta \eta$ in time and space respectively. The modified Eqs. (13)-(16) are integrated from $t=0$ to $t=1$. Then, the solution obtained at $t=1$ is used as the initial condition for integrating Eqs. (5)-(7) and (11) from $t=1$ up till the steady state.

The resulting system of equations has to be solved in the infinite domain $0<\zeta<\infty$. A finite domain in the $\zeta$-direction can be used instead with $\zeta$ chosen large enough to ensure that the solutions are not affected by imposing the asymptotic conditions at a finite 
Military Technical College

Kobry Elkobbah,

Cairo, Egypt

May $29-31,2012$

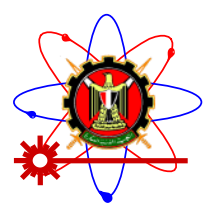

$6^{\text {th }}$ International Conference

on Mathematics and

Engineering Physics

(ICMEP-6)

distance. The independence of the results from the length of the finite domain as well as the grid density was ensured and successfully checked by various trial and error numerical experimentations. Computations are carried out for $\zeta_{\infty}=10$ and step size $\Delta \zeta=0.04$ which are found adequate for the ranges of the parameters studied here. Larger finite distance or smaller step size do not show any significant change in the results. Convergence of the scheme is assumed when all of the variables $F, G, H, \theta, \partial F / \partial \zeta, \partial G / \partial \zeta$, and $\partial \theta / \partial \zeta$ for the last two approximations differs from unity by less than $10^{-6}$ for all values of $\zeta$ in $0<\zeta<10$ and all $t$.

\section{Results and Discussion}

The evolution of the axial velocity component at infinity $H_{f}$ for $K=0$ and $M=0$ is shown in Fig. 1a for various values of the parameter $S$. It is clear that increasing the suction velocity increases the axial velocity towards the disk and decreases its growth time up to the steady state. For small values of the suction parameter, the effects of the radial flow in addition to the suction through the disk help to increase the quantity of fluid drawn with time. Then, two paths are available for the incoming fluid, one through the suction holes of the disk and another in the radial direction. Higher values of the suction velocity provide an easier path for the flow through the wall than that in the radial direction and result in an almost time-independent inflowing stream towards the disk. For the case of injection, $H_{f}$ initially equals the injection velocity and for few radians of the rotation of the disk it keeps its positive direction. With time progression, the radially ejected fluid pumps the flow towards the disk and a crossover point in time that depends on the injection parameter $S$ occurs and the axial flow reverses direction till its steady state value. The greater the injection velocity the more strongly the inflow opposed and, consequently, the later appearance of the crossover of the axial velocity. Notice that, the axial velocity towards the disk increases monotonically with time. It is also depicted that the fluid injection at the disk results in the familiar inflection-point shapes, especially for large values of the injection parameter $S$. Therefore it is expected that high injection velocities tend to destabilize the laminar flow and lead to transition to turbulence. 
Military Technical College

Kobry Elkobbah,

Cairo, Egypt

May $29-31,2012$ $6^{\text {th }}$ International Conference

on Mathematics and

Engineering Physics

(ICMEP-6)

Figure $1 \mathrm{~b}$ indicates the effect of the non-Newtonian fluid characteristics on $H_{f}$ for different values of the parameter $S$ with $K=1$ and $M=0$. The figure shows that $H_{f}$ overshoots for all suction or injection velocities. The overshooting is more pronounced for the case of injection than suction and it increases with the increment in the parameter $S$ while the time at which it occurs decreases. Comparison between Figs. 1a and $1 \mathrm{~b}$ indicates that the time of appearance of the crossover points in the case of injection increases with increasing $K$. It is also shown in Fig. $1 \mathrm{~b}$ that in the case of zero velocity at the disk $(S=0)$, there is a reversal in the direction of $H_{f}$ and a crossover in time occurs in this case. The parameter $K$ tends generally to resist the axial flow towards the disk and reduces its velocity and growth time. It is evident from the figure that increasing $K$ gives rise to the inflection-point shapes of $H_{f}$ with time for all values of the injection velocities and even in the case of zero injection velocity. Consequently, the increment in $K$ is expected to destabilize the laminar flow. Notice that, in the case of large suction velocities, the incoming flow velocity reaches a steady state value lower than that at the disk. It is also clear that for large values of time, the parameter $K$ has more apparent effect on $H_{f}$ in the case of suction more than that in the case of injection.

Figure 1c indicates the effect of the porosity on $H_{f}$ for different values of the suction or injection parameter $S$ with $K=0$ and $M=1$. The porosity leads generally to a reduction in the magnitude of the axial velocity at infinity and its growth time. For the case of uniform suction, the porosity has an apparent effect on the flow for small values of the suction parameter while its effect becomes negligible for large values of $S$. In the case of uniform injection, the porosity restrains the azimuthal and radial flows and then supports the out flowing stream of injected flow to stop completely the drawn inflow and prevent the occurrence of the crossover points during time progression. It is clear that the porosity has an apparent effect in controlling the shape of the inflection-point profiles in the case of high injection velocities. Consequently, the porosity works to stabilize the laminar flow and stops the transition to turbulence. With porosity, varying the injection velocity becomes more effective on the flow than in the non-porous case. The porosity 
Military Technical College

Kobry Elkobbah,

Cairo, Egypt

May $29-31,2012$

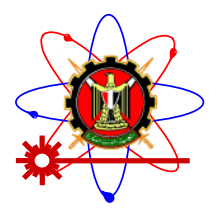

$6^{\text {th }}$ International Conference

on Mathematics and

Engineering Physics

(ICMEP-6)

has a marked effect on the flow for the whole range of the injection velocities. However, its effect is much lower for small suction velocities and almost neglected for higher suction velocities.

Figure 1d indicates the evolution of $H_{f}$ for various values of the parameter $S$ with $K=1$ and $M=1$. The reduction in $H_{f}$, the appearance of the overshoots (as an effect of $K$ ) as well as the suppression of the crossover points in the case of injection (as an effect of the porosity $M$ ) are shown in the figure. However, the most interesting result is the reversal of the direction of the axial flow with time progression up till the steady state in the case of $S=0$. The combined effect of the porosity and the non-Newtonian fluid characteristics results in closing the two paths for the axial flow towards the disk and then reverses its direction.

Figure 2a presents the time development of the Nusselt number $N u$ for different values of the suction or the injection velocities with $K=0, M=0$ and $\operatorname{Pr}=0.7$. It is clear that the effect of the fluid injection is to decrease the heat transfer significantly (and, hence, the Nusselt number) by blanketing the surface with fluid whose temperature is close to $T_{w}$. Suction has an opposite effect on the heat transfer, since fluid at nearambient temperature is brought to the neighborhood of the disk surface. Figure $2 \mathrm{~b}$ presents the time development of the Nusselt number $N u$ for different values of the suction or the injection velocities with $K=1, M=0$ and $P r=0.7$. It indicates that, for all values of suction or injection velocities, increasing $K$ decreases $N u$ due to its effect in decreasing the axial velocity towards the disk. Figure $2 \mathrm{c}$ presents the time development of the Nusselt number $N u$ for different values of the suction or the injection velocities with $K=0, M=1$ and $\operatorname{Pr}=0.7$. Figure 2c shows that increasing $M$ decreases $N u$ due to its effect in restraining the axial flow towards the disk. Figure $2 \mathrm{~d}$ presents the time development of the Nusselt number $N u$ for different values of the suction or the injection velocities with $K=1, M=1$ and $\operatorname{Pr}=0.7$. Figure $2 \mathrm{~d}$ indicates the same findings in Figs. 1c and $1 \mathrm{~d}$.

Figures $3 \mathrm{a}, \mathrm{b}, \mathrm{c}, \mathrm{d}$ present the steady state velocity components and temperature, $F$, $G, H$, and $\theta$, respectively, for various values of $K$ and for $M=0$ and 1 . In these figures, 
Military Technical College

Kobry Elkobbah,

Cairo, Egypt

May 29-31,2012

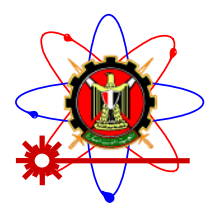

$6^{\text {th }}$ International Conference on Mathematics and Engineering Physics

(ICMEP-6)

$S=0$ and $P r=0.7$. It is clear from Fig. 3a that increasing $K$ increases $G$ for all $\zeta$. Figure 3a shows also the damping effect of the porosity which results in a reduction in the velocity component $G$ for all $\zeta$. Figure $3 b$ indicates that, for $M=0$, increasing the parameter $K$ decreases $F$ for small and moderate values of $\zeta$. However, for larger values of $\zeta$ a crossover point that depends on $K$ appears and an increment in $K$ increases $F$. Figure $3 \mathrm{~b}$ presents an interesting effect for the porosity in the suppression of the crossover points that appear with the variation of $K$. Also, it is shown that the influence of the porosity, for large values of $K$, in reversing the direction of the velocity component $F$ with $S=0$. The porosity has the effect in reducing the magnitude of $F$ for all $\zeta$ and for various values of $K$. Figure $3 \mathrm{c}$ shows that increasing the parameter $K$ or $M$ increases the resistance for the incoming axial flow and consequently reduces the axial velocity towards the disk $H$ for all $\zeta$. For large values of $K$, due to the reversal of the direction of $F$ which is the source for $H$, a resistance is imposed on the axial flow towards the disk and consequently the direction of $H$ is reversed. Figure $3 \mathrm{~d}$ indicates that increasing $K$ or $M$ increases $\theta$ for all $\zeta$ due to the effect of $K$ or $M$ in damping $H$ which prevents bringing the fluid at a near-ambient temperature towards the surface of the disk. It is noticed that, for large values of $K$, the combined effect of $K$ and $M$ results in the diminishing of the effect of the term $H \partial \theta / \partial \zeta$ and then leads to a linear dependence of $\theta$ on $\zeta$.

Figure 4a presents the influence of the axial flow at the surface of the disk on the steady state radial velocity profile $F$ for the case of suction or injection with $K=0$ and $M=0$. It is clear that increasing the suction velocity leads to a rapid decrease in $F$ while increasing the injection velocity increases $F$. Figure $4 \mathrm{~b}$ presents the steady state radial velocity profile $F$ for various values of suction or injection velocities and for $K=0.5$ and $M=0$. A comparison between Figs. $4 \mathrm{a}$ and $4 \mathrm{~b}$ shows that, for the whole range of injection velocities and small suction velocities, increasing $K$ decreases $F$ for all $\zeta$. When the suction velocity is large, increasing the parameter $K$ reverses the direction of $F$. With $K=0.5$ increasing the suction velocity leads to an increment in the magnitude of $F$ for 
Military Technical College

Kobry Elkobbah,

Cairo, Egypt

May $29-31,2012$

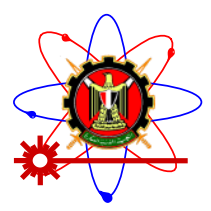

$6^{\text {th }}$ International Conference

on Mathematics and

Engineering Physics

(ICMEP-6)

small $\zeta$ while decreases its magnitude as $\zeta$ increases which results in the appearance of crossover points with $\zeta$. The distance from the disk at which the crossover appears decreases with increasing the suction velocity. This is due to the fact that increasing the suction velocity pumps the reversed flow in the negative radial direction near the disk and increases its velocity. At greater distances from the disk, increasing suction helps the axial flow towards the disk which stops the reversed radial flow and then decreases its velocity. The influence of the porosity on the steady state profiles of $F$ is shown in Fig. $4 \mathrm{c}$ with $K=0$ and $M=1$ and for various values of the parameter $S$. The porosity effect is to sustain the flow in the radial direction for all values of the suction or injection velocities. However, its effect is more pronounced in the case of injection more than that in the case of suction.

Figure $4 \mathrm{~d}$ presents the steady state radial velocity profile $F$ for various values of suction or injection velocities and for $K=0.5$ and $M=1$. Increasing the two parameters leads to a great reduction in $F$ for all values of the injection velocities. In the case of suction, the parameters $K$ and $M$ result in reversing the direction of $F$ and increasing its magnitude for all values of the suction velocity. The figure also presents the appearance of the crossover points in $F$ profiles due to changing the parameter $S$.

Figure 5a presents the influence of the axial flow at the disk surface on the steady state axial velocity profile $H$ for the case of suction or injection with $K=0$ and $M=0$. Increasing the suction velocity increases the axial flow towards the disk for all $\zeta$ while the magnitude of the axial velocity at infinity is larger than that at the disk. Increasing the injection velocity reduces the axial flow towards the disk. With increasing injection velocity, the outflow penetrates to greater distances from the disk surface. Consequently, the crossover point between the positive and negative axial velocity is pushed farther outward in the $\zeta$-direction. It is seen also that the fluid injection gives rise to the familiar inflection-point profiles, especially for high values of the injection parameter $S$. Then, high injection velocities are expected to destabilize the laminar flow and lead to transition to turbulence. Figure $5 \mathrm{~b}$ shows the steady state axial velocity profile $H$ for 
Military Technical College

Kobry Elkobbah,

Cairo, Egypt

May $29-31,2012$ $6^{\text {th }}$ International Conference

on Mathematics and

Engineering Physics

(ICMEP-6)

different values of the parameter $S$ with $K=1$ and $M=0$. Increasing the parameter $K$ decreases the axial flow towards the disk for all values of the parameter $S$. In the case of suction, for $K=1$, the magnitude of the axial velocity at infinity is smaller than that at the disk. The crossover points appear in $H$ profiles are pushed further in the $\zeta$-direction when increasing $K$. Figure 5c presents the steady state axial velocity profile $H$ for various values of the parameter $S$ with $K=0$ and $M=1$. The porosity effect is to sustain the flow in the axial direction. It leads to a reduction in the axial flow towards the disk for small suction velocities and its effect becomes negligible for larger suction velocities. With injection applied at the disk, the increasing the porosity parameter decreases the azimuthal and radial flows and, consequently, the injection stream sustains its axial motion towards the disk. It is also seen in the figure, the influence of the porosity in the suppression of the crossover of the axial component of velocity and then the reversal of the direction of the axial motion. It is clear that the porosity has a marked effect in changing the shape of the inflection-point profiles in the case of high injection velocities. Consequently, the porosity works to stabilize the laminar boundary layer and prevents the transition to turbulence. Figure $5 \mathrm{~d}$ presents the steady state axial velocity profile $H$ for different values of the parameter $S$ with $K=1$ and $M=1$. Introducing the two parameters $K$ and $M$ results in the reversal of the direction of the axial flow for $S=0$ and in the suppression of the crossover points of $H$ profiles in the case of injection.

\section{Conclusions}

In this study the time varying flow of a non-Newtonian fluid induced by a rotating disk with heat transfer in a porous medium was studied in the presence of uniform suction and injection. The effect of the porosity of the medium, the non-Newtonian fluid characteristics and the uniform suction or injection velocity on the velocity and temperature distributions was considered. It is shown that the porosity results in the suppression of the crossover points of the axial component of velocity in the non-porous case with uniform injection, while the non-Newtonian parameter $K$ leads to the earlier time appearance of these crossover points. The parameter $K$ has an interesting effect on 
Military Technical College

Kobry Elkobbah,

Cairo, Egypt

May $29-31,2012$

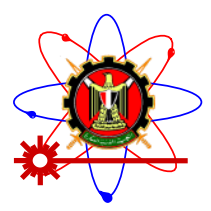

$6^{\text {th }}$ International Conference

on Mathematics and

Engineering Physics

(ICMEP-6)

reversing the direction of the axial velocity at infinity during time even in the case of zero suction velocity and in the non-porous case. The inclusion of the porosity, with the nonNewtonian behavior of the fluid, results in reversing the direction of the axial velocity for $S=0$ for all time. Another interesting result is the effect of the parameter $K$ in reversing the direction of the radial velocity for large suction velocities in the non-porous case. The combined effect of both parameters $K$ and $M$ has an interesting feature in reversing the direction of the radial flow for all suction velocities and even in the case of zero suction velocity. One more effect for the porosity is to suppress the crossover occurs in the radial velocity profiles due to the variation of $K$. The porosity works to stabilize the flow while the non-Newtonian fluid characteristics tend to destabilize the flow.

The porosity has a more pronounced effect on the flow in the case of injection than suction and its effect can be neglected for large suction velocities. On the other hand, the non-Newtonian characteristics has an apparent effect on the flow for all values of suction or injection velocities while its effect on the flow in the case of suction is more pronounced than the case of injection. Therefore, the two parameters $K$ and $M$ together have an apparent effect on the flow for the whole range of the axial velocity at the surface of the disk. The non-Newtonian fluid characteristics leads generally to a reduction in the heat transfer from the disk for all suction or injection velocities, while the effect of the porosity on the heat transfer can be neglected.

\section{References}

1. von Karman, T., "Uber laminare und turbulente reibung", ZAMM, vol. 1, No. 4, pp. 233-235, 1921.

2. Cochran, W.G., "The flow due to a rotating disk", Proc. Cambridge Philos. Soc. 30 (3), pp. 365-375, 1934.

3. Benton, E.R., "on the flow due to a rotating disk", Fluid Mechanics, Vol. 24, No. 4, pp. 781-800, 1966.

4. Millsaps, K. and Pohlhausen, K., "Heat transfer by laminar flow from a rotating disk", J. of the Aeronautical Sciences, Vol. 19, pp. 120-126, 1952.

5. Sparrow, E.M. and Gregg, J.L., "Mass transfer, flow, and heat transfer about a rotating disk", ASME J. of Heat Transfer, pp. 294-302, Nov. 1960.

6. Attia, H.A., "Unsteady MHD flow near a rotating porous disk with uniform suction or injection", Fluid Dynamics Research 23, pp. 283-290, 1998. 
Military Technical College

Kobry Elkobbah, Cairo, Egypt May 29-31,2012

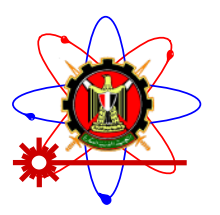

$6^{\text {th }}$ International Conference on Mathematics and Engineering Physics

(ICMEP-6)

7. Mithal, K.G., "On the effects of uniform high suction on the steady flow of a nonNewtonian liquid due to a rotating disk", Quart. J. Mech. Appl. Math., XIV, pp. 401-410, 1961.

8. Attia, H.A., "Unsteady flow of non-Newtonian fluid above a rotating disk with heat transfer", International Journal of Heat and Mass Transfer, 46, pp. 2695-2700, 2003.

9. Attia, H.A., "On the effectiveness of uniform suction and injection on unsteady rotating disk flow in porous medium with heat transfer", Computational Materials Science, Elsevier Science, Ltd, Vol. 38, pp. 240-244, 2006.

10. Ingham, D.B. and Pop, I., "Transport phenomena in porous media", Pergamon, Oxford, 2002.

11. Khaled, A.R.A. and Vafai, K., "The role of porous media in modeling flow and heat transfer in biological tissues", Int. J. Heat Mass Transf., 46, pp. 4989-5003, 2003.

12. Ames, W.F., Numerical methods in partial differential equations, 2nd ed., Academic Press, New York, 1977. 